\title{
Automatic Detection of Liver in CT Images using Optimal Feature based Neural Network
}

\author{
Ritu Punia \\ Department of Computer Science and Engineering \\ PEC University of Technology \\ Chandigarh, INDIA
}

\author{
Shailendra Singh \\ Department of Computer Science and Engineering \\ PEC University of Technology \\ Chandigarh, INDIA
}

\begin{abstract}
This paper presents a method for automatic detection of liver in CT images using optimal texture features. As image contains noise so firstly, image is pre-processed with median filter. Regions of interests are chosen carefully from both liver and non-liver areas. Texture features are extracted from selected regions of interest using first order statistics and wavelet transform. Neural Network is used for classification of pixels into liver and non-liver areas. Accuracy of classification process depends on number of features extracted which should be chosen carefully. For careful selection of features, optimal features are selected from extracted features using genetic algorithm and used for final classification of image pixels. The method is tested on CT images and results obtained are presented both qualitatively and quantitatively.
\end{abstract}

\section{Keywords}

Liver Detection, Texture, Optimal Features, Genetic Algorithm, Neural Network.

\section{INTRODUCTION}

Liver detection in computed tomography images has become very important these days because it is first and foremost task before diagnosing liver diseases like liver tumor, 3D liver volume rendering and liver transplantation. Liver image segmentation is very difficult task as neighbouring organs have similar pixel intensity as of liver. Liver can be segmented manually from CT images but manual segmentation varies from person to person. As intensity of pixels of other organs is similar to liver pixels, various researchers have utilized texture features in combination with machine learning techniques and provided automated mean for segmenting liver from CT images. First attempt of this type using neural network was made by Tsai and Tanahashi [1]. Histogram based features are extracted from $7 \times 7$ pixel region and fed as input to feed-forward neural network which is trained with back-propagation. It classifies pixels into three classes; liver pixels, boundary pixels and non-liver pixels. This method needs large training set to learn variability among different patients. To overcome large training set problem, Koss et al. [2] utilized unsupervised learning method that is Hopfield neural network and haralick [3] texture features. To gain clearer picture of liver area, five histogram based features are extracted from $5 \times 5$ pixel window and used as input to multi-layer feed forward network which is trained with back-propagation [4]. It classifies pixels two classes as belonging to either liver area or non-liver.

Watershed transform combined with neural network is utilized in [5]. Image is pre-processed using morphological operations (morphological smoothing, intensity threshold and morphological gradient). Watershed transform is used to partition gradient image. The feed forward neural network trained with back propagation algorithm is used to liver features extraction in training phase. These features are used in liver recognition. This work is extended in [6] by using combination of iterative watershed and neural network. In this method, after applying morphological pre-processing, six shape based features are extracted from 10 biggest regions using both watershed and multi-layer feed forward iteratively and compared and the error is used to adjust required parameters automatically.

Other machine learning techniques like support vector machine have been used in literature also for automatic detection of liver. Wavelet transform [7] based features are extracted from input abdominal image and provided as input to support vector machine which classifies pixels into liver and non-liver pixels and morphological operations are used to refine results [8]. Performances of haralick texture features and wavelet transform features are compared in [9]. Wavelet transform for feature extraction, support vector machine for classification and region growing for post-processing is used in [10].

This paper is organized as follows: section 2 gives outline of proposed method; section 3 describes different phases of system development, section 4 presents results obtained and finally section 5 concludes the paper.

\section{SYSTEM OUTLINE}

The proposed method provides automatic (where no user interaction is required) solution to the liver CT image segmentation problem. In this method, firstly image is preprocessed. Pre-processing step consists of three parts. Firstly, image is cropped based on prior knowledge of liver location in abdominal CT images. Usually, liver is present in upper left side and lower right side does not contain liver. So, image is cropped such that extra right part is cropped automatically without cropping any liver part. $3 \times 3$ median filter is applied on cropped image. Image is sharpened to enhance quality of edges just for eye visualization without adding new details. After successfully completing image pre-processing, histogram based features and wavelet transform based features are extracted from region of interests. Texture Feature extraction is used because simple intensity based features cannot be used since neighbouring pixels also have similar pixel intensity as of liver while texture of different regions is different. Optimal features are selected and used as input to the classifier because original feature set may contain redundant and noisy feature which affects the segmentation process. Genetic algorithm is used for optimal feature selection because it provides global optimization. During classification process, feed-forward neural network trained with back propagation is used. Next subsections provide 
details of different techniques used in overall system development.

\subsection{Feature Extraction}

In medical imaging, local features are more meaningful than single pixels [11]. Feature extraction plays an important role in pattern recognition especially in liver image segmentation because intensity of neighbouring pixels is similar to liver region which makes it difficult to segment liver from CT images using gray level intensity. As texture of different regions of an image is different so texture features are extracted from pre-processed CT images. Feature extraction is defined as task of developing a set of features from an image and classifying image regions based on these texture features. The extraction of set of features creates a feature vector which takes the form: $x=\left[x_{1}, x_{2}, \ldots, x_{n}\right]$. First order Histogram based features and wavelet transform based features, are extracted from region of interest. For training, these regions of interest are selected manually from liver and non-liver regions. Six histogram based features and twenty eight wavelet transform based features are extracted from $5 \times 5$ window size [4] from liver and non-liver region both. Details of different features are explained below

\subsubsection{First Order Histogram Based Features}

First order statistical features are the simplest. These are computed from histogram of the image. The histogram of a digital image with intensity levels $[0, \mathrm{~K}-1]$ is defined as follows [12]:

$$
H(k)=\frac{n_{k}}{M N} ; k=0,1, \ldots ., K-1
$$

where $\mathrm{M}$ and $\mathrm{N}$ are the rows and column dimensions of the image, $\mathrm{K}$ is maximum number of gray levels and $n_{k}$ is the number of pixels in the image with intensity k. Roughly speaking, histogram measures the probability of occurrence of certain pixel in an image.

As histogram is probability function of pixel values, therefore it can be used for determining a set of statistical parameters [13] known as first order statistics. Histogram based features described are listed below:

$$
\begin{aligned}
& \text { Mean: } \quad x_{1}=\sum_{k=0}^{K-1} k H(k) \\
& \text { Variance: } \quad x_{2}=\sum_{k=0}^{K-1}\left(k-x_{1}\right)^{2} H(k) \\
& \text { Standard Deviation: } \quad x_{3}=\sqrt{x_{2}} \\
& \text { Skewness: } \quad x_{4}=x_{3}{ }^{-3} \sum_{k=0}^{K-1}\left(k-x_{1}\right)^{3} H(k) \\
& \text { Kurtosis: } \quad x_{5}=x_{3}{ }^{-4} \sum_{k=0}^{K-1}\left(k-x_{1}\right)^{4} H(k) \\
& \text { Entropy: } \quad x_{6}=\sum_{k=0}^{K-1}[H(k)]^{2}
\end{aligned}
$$

The mean is a measure of average level of intensity of image. The variance measures variation of intensity around mean. Skewness is a measure of symmetry of histogram. If histogram is symmetrical around mean then skewness is zero and otherwise it is positive or negative if it is skewed above or below the mean. The kurtosis describes the flatness of histogram. The entropy is a statistical measure of randomness of histogram.

\subsubsection{Wavelet Transform Based Features:}

The discrete wavelet transform (DWT) is defined as decomposition of as signal $f(t) \in L^{2}(R)$ into a family of functions $\psi_{m, n}(t)$ obtained through translation and dilation of a kernel function $\psi(t)$ known as mother wavelet [14]:

$$
\psi(t)=2^{-m / 2} \psi\left(2^{-m} t-n\right)
$$

where $\mathrm{m}$ and $\mathrm{n}$ are scale and translation indices respectively.

In case of two-dimensional images, the wavelet decomposition is obtained by applying separate filters along the rows and along the columns of an image [14]. One-scale image decomposition using wavelet transform (see figure 1(a)) results in four blocks of components: First block (LL) represents the downsampling of low-pass filter along both rows and columns; second block (LH) represents the downsampling of the low-pass filter along rows and high-pass filter along columns; third block (HL) represents the downsampling of the high-pass filter along rows and low-pass filter along columns; and fourth block $(\mathrm{HH})$ represents the downsampling of the high-pass filter along both rows and columns. Such filtering or decomposition can be done further on LL, which results in a higher scale representation of the original image (see figure 1(b)).

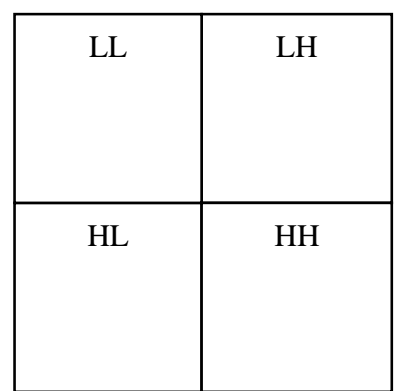

(a)

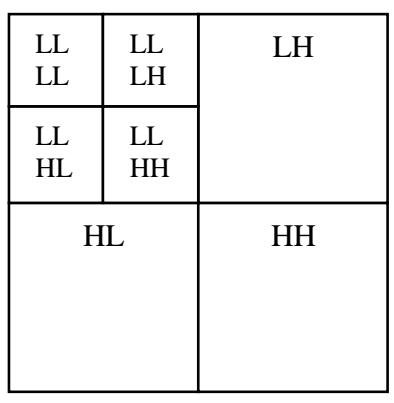

(b)
Figure 1: (a) 1 Scale Image Decomposition, (b) 2 Scale Image Decomposition

Various statistical features can be extracted after decomposing image using wavelet transform. Energy, mean, standard deviation [15] have been used in proposed method for creating feature vector.

Energy:

$$
e_{n}=\frac{1}{N^{2}} \int\left|W_{n}(x, y)\right| d x d y
$$

Mean:

$$
\mu_{n}=\int\left|W_{n}(x, y)\right| d x d y
$$

Standard Deviation: 


\subsection{Genetic Algorithm Based Feature Selection}

Many pattern recognition tasks like medical diagnosis systems require learning of an appropriate classification function that assigns a given input pattern (represented by a set of features for that pattern) to one of a finite set of classes. The choice of these features used to represent input patterns affect:

- The accuracy of the classifier used for classification of patterns. If numbers of features used for representation of input patterns are not enough to describe useful properties of the pattern then it may result in nonaccurate classification of patterns.

- The time needed for classification function to learn features representing input patterns. The features representing input patterns determine search space that need to be explored by classification function in learning. An abundant of irrelevant features can unnecessarily increase the size of search space and hence the time required for learning.

- The cost of classification process using the learned classification function.

Due to above mentioned reasons [16], optimal selection of features has become an important step in pattern recognition problems. Thus, feature selection refers to the task of selecting useful and optimal subset of features to be used to represent input patterns with high accuracy, reduced time and cost of classification function.

Feature selection is broadly classified in two groups namely filter and wrapper approach. The filter approach does not depend on classification process. Time taken for this approach is less but results of classification are not very much accurate. The wrapper approach depends on classification process. Wrapper approach has been used in proposed method. The genetic parameters used in proposed method are explained below:

Each region of interest is represented as feature vector. A set of 34 features are extracted per pixel. In proposed encoding scheme, one bit is used to represent each feature. If the value of bit is 1 , feature corresponding to this bit is selected otherwise it is not selected.

Table 1. Genetic Parameters

\begin{tabular}{|l|l|}
\hline Genetic Parameters & Value \\
\hline Population Size & 80 \\
\hline Population Type & bitString \\
\hline Number of Variables & 34 \\
\hline Fitness Function & Mean Square Error \\
\hline Selection Function & Roulette Wheel \\
\hline Crossover Function & Two Point Crossover \\
\hline Mutation Function & Gaussian Mutation \\
\hline
\end{tabular}

As wrapper approach is used for evaluation of individuals, so fitness function depends on classifier. Simple fitness function is used that is the performance of neural network classifier.
Mutation and crossover are two of the most commonly used operators with genetic algorithm that represent individuals as binary strings. Mutation operates on a single string and generally changes a bit at random. Crossover operates on two parent strings to produce two offspring. Genetic Parameters are summarized in above table.

\subsection{Neural Network Classifier}

Multi-layer feed forward neural network is used as a classifier for classification of pixel as belong to liver or non-liver pixels. Neural network consists of three layers. First is the input layer. Second is the hidden layer. Hidden layer consists of 10 neurons. The third or output layer consists of one neuron representing output. 34 features are fed as input to the input layer of neural network. The network is trained with Levenberg-Marquardt back-propagation learning algorithm. It is the fastest learning algorithm. Output layer has only one output and it represents whether the input pixel belongs to liver region or not. If output is 1 , the corresponding input pixel is said to belong to liver region and output for non-liver pixels is represented by 0 . Figure 2 shows schematic diagram of neural network.

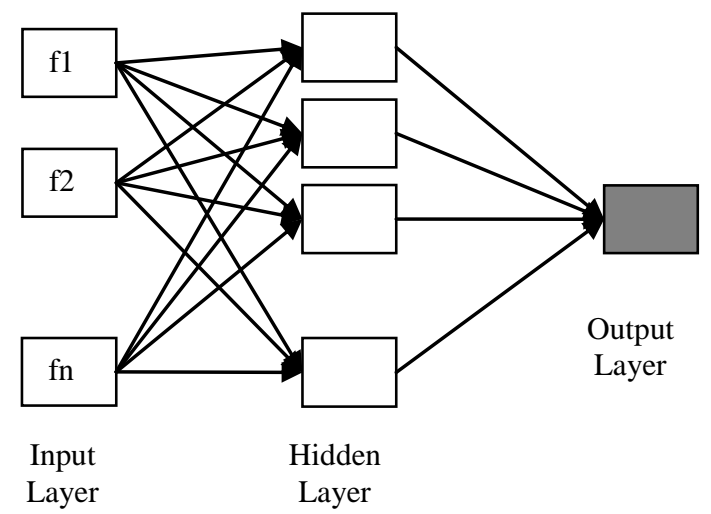

Figure 2: Schematic Diagram of Back Propagation NN

\subsection{Post Processing}

Neural Network classifier here used in a pixel based manner i.e., output is returned pixel by pixel. Output corresponding to liver pixels is set to 1 and output for pixels belonging to nonliver is set to 0 . This output returned by neural network need to be refined because it may be possible that some liver pixels are detected as belonging to non-liver and set to 0 and some non-liver pixels are detected as belonging to liver pixels and set to 1 . So, to refine these results post-processing is needed. As liver is the largest area in abdominal CT image, so during post-processing, firstly, the largest area is extracted from image returned by classifier. For filling holes in liver regions and removing any extra part morphological operations (open and close) are used.

\section{SYSTEM DEVELOPMENT}

The system is developed in four stages:

$\begin{array}{ll}\text { a. } & \text { Training Mode } \\ \text { b. } & \text { Feature Optimization Mode } \\ \text { c. } & \text { Testing Mode } \\ \text { d. } & \text { Function Mode }\end{array}$


A set of ten CT images is used in training mode. Same set of ten images is used for feature optimization mode. Another set of ten images is used during testing mode. Finally graphical user interface was developed for user mode that is function mode.

\subsection{Training Mode}

The training set consists of ten CT images. This set is used for supervised training of neural network. Regions of interest are extracted manually from liver and non-liver areas of these images and saved. These regions are selected all over the liver region with slight overlapping to get complete representation. Six histogram based features and twenty eight wavelet transform based features are extracted from both liver and non-liver regions. Thus, 34 features are calculated and stored in a feature vector $\mathrm{f}(\mathrm{x}, \mathrm{y})=(\mathrm{f} 1, \mathrm{f} 2, \mathrm{f3} \ldots . . \mathrm{f} 34)$. These features are calculated over a $5 \times 5$ window. 500 training patterns are randomly chosen from each of liver and non-liver regions of each image. Thus training dataset consists of $10000 \times 34$ feature matrix. The system output is 1 for liver region and 0 for all other regions. During training mode, neural network is trained for complete feature set.

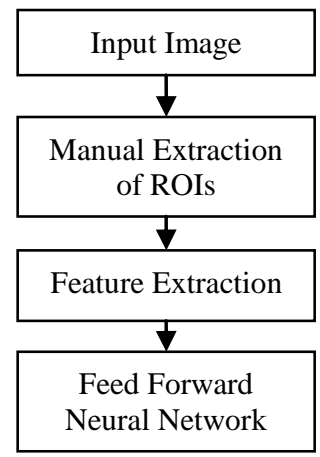

Figure 3: Block Diagram of Training mode

\subsection{Feature Optimization Mode}

After successful training of complete feature set, feature optimization mode starts. The task of feature optimization mode is to select fewer and optimal features from complete feature set. Wrapper approach of feature selection algorithm has been used in proposed method. In wrapper approach, fitness function depends on learning algorithm used. So feature optimization process is combination of both genetic and neural network. Mean Square Error of neural network performance has been used as fitness function for evaluation criteria. Genetic based feature selection can be summarized as follows:

i. Initialize the first population; population type used here is bit string.

ii. Begin evolution loop until the stopping criterion is reached.

iii. For each chromosome in the population, multiply each chromosome value with the feature value and compute new individuals.

iv. For each individual calculate fitness function.

Apply genetic operators; selection, crossover, mutation to form new generation.

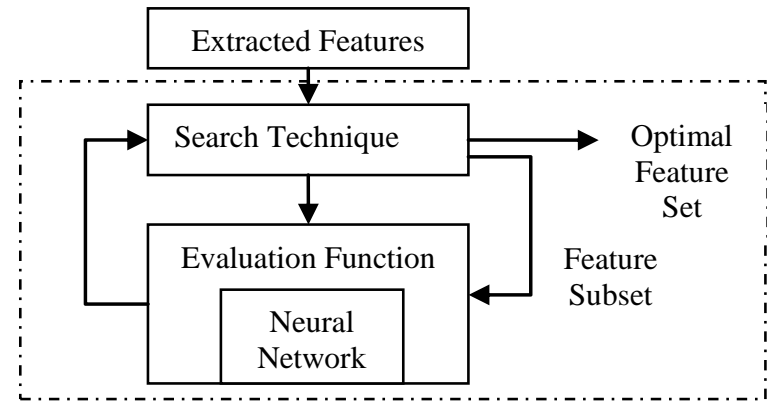

Figure 4: Genetic Algorithm Based Feature Selection

\subsection{Testing Mode}

After successful completion of genetic optimization of feature set, testing takes place. In testing mode, test data set is composed of similar to the training but from different CT images. But during testing mode, only the optimal features returned from optimization mode are used. This data set is tested on trained genetic based neural network.

\subsection{Function Mode}

After successful testing of neural network; when network gives acceptable result, the system is ready for function mode i.e., user mode. In this mode a graphical user interface is made for ease of user. During function mode, system automatically detects liver in CT image. Function mode starts when user inputs an image to the system. Then, input is pre-processed based on the prior knowledge of location of liver and noise is removed and for enhancing edge quality image is sharpened. After pre-processing of original image, optimal features, returned by genetic feature selection algorithm, are calculated pixel by pixel over the entire image. These features are calculated in $5 \times 5$ neighbourhood sliding over the image. Features returned by this block are assigned to the pixel which is the centre point of this block. Features are stored in feature vector pixel by pixel.

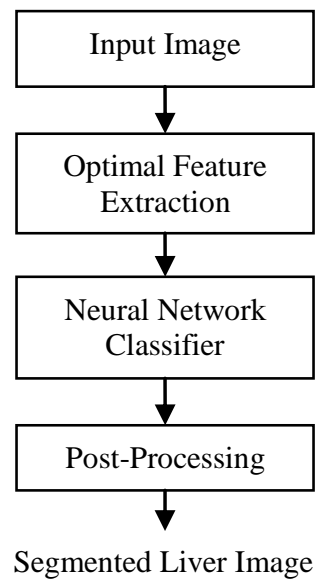

Figure 5: Block Diagram of Function Mode

Complete feature matrix of image is then input to the trained neural network. The output for a pixel which belongs to liver region is unity and output for the pixel belonging to non-liver region is set to zero. The output image returned by neural network need to be refined because some liver pixels may be detected as non-liver pixels and some non-liver pixels may be detected as liver pixels. So, for final segmentation, post- 
processing is applied to the image returned by classifier. Final automatic process in user mode for liver detection is shown in figure 5 .

\section{RESULTS AND COMPARISON}

In this paper, an automatic method for detecting liver in CT images is provided. During function mode, CT images are used to analyze the performance of proposed method. The images are collected by searching for liver CT images on different sites [17]. MATLAB 7.11 is used for implementation. The method classifies image pixels into one of two classes; liver or non-liver. It goes through a series of steps. At each step, system displays some output. These stepwise outputs are shown differently section wise below.

\subsection{Pre-Processing Results}

During pre-processing step, firstly image is cropped for reducing the computational burden. Then median filter is applied to denoise the image which is followed with the application of image sharpening filter which is used to enhance edge qualities.

\subsection{Genetic Optimization Results}

This section provides firstly the results obtained by applying genetic optimization to extracted features. Then it shows graph of optimal features of both liver and non-liver areas.

Genetic parameters are set prior to execution of genetic algorithm. Various variables are set; these are population type, population size, number of variables, selection function, crossover function and mutation function. Numbers of variables are equal to number of extracted features which are 34 and initial population is generated randomly. Various Parameters that are used are described in table 1 . When the genetic optimization function is run, it plots best fitness function and it returns optimal features. Total numbers of features are 34 and optimal features returned by genetic algorithm are 13.
Genetic algorithm selects optimal features. Numbers of optimal features selected by genetic algorithm are 13. Four first order histogram based features are selected out total six features. Nine wavelet transform based features are selected out of total 28 features.

Optimal Feature Set:

1101010010000100100000100010110101

The variables which are set to 1 are selected in feature extraction process and the variables which are set to 0 are not selected.

\subsection{Neural Network Results}

After optimizing the features, the optimal features are input to trained neural network which classifies pixels based on their feature values. During training of neural network all the features are used for training. But for final classification of input image, only the optimal features are considered values of all the remaining features are set to 0 . Feed-forward neural network is used for classification which is trained using backpropagation learning algorithm.

Neural network has three layers: Input layer takes 34 features as input, hidden layer has 10 neurons. 10 neurons are selected after careful inspection by taking different number of neurons and output with 10 neurons is acceptable as compared to more neurons. Net has only one output in output layer. Output for a feature is 1 or 0 based on feature value. Output is set to 1 for liver pixels and output is 0 for non-liver pixels.

\subsection{Post Processing Results}

Output of neural network is not perfect. It needs some refinement because some pixels are wrongly classified. Some pixels within liver region are classified as non-liver pixels and some non-liver pixels are classified as liver pixels. So, some post-processing is needed for getting clear picture of liver. 


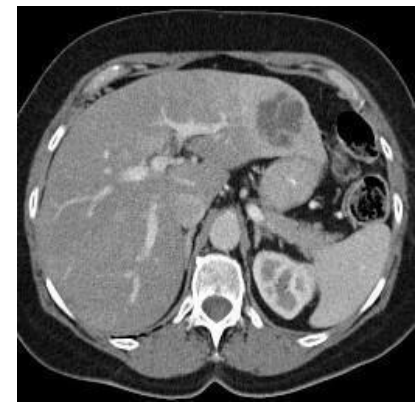

(a)

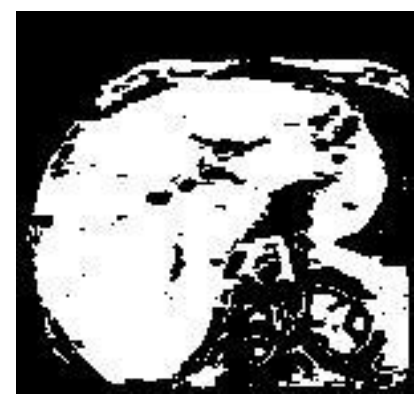

(d)

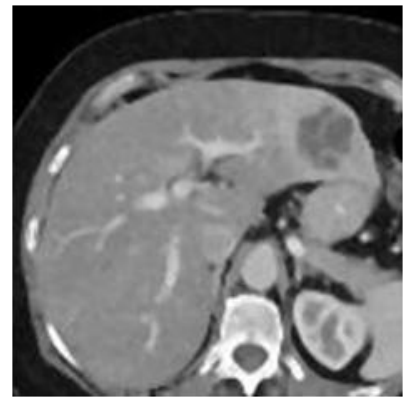

(b)

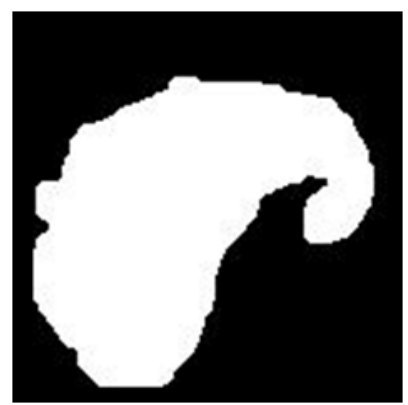

(e)

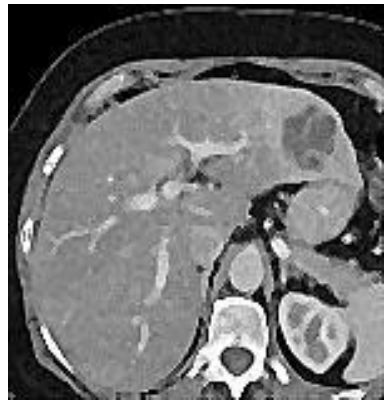

(c)

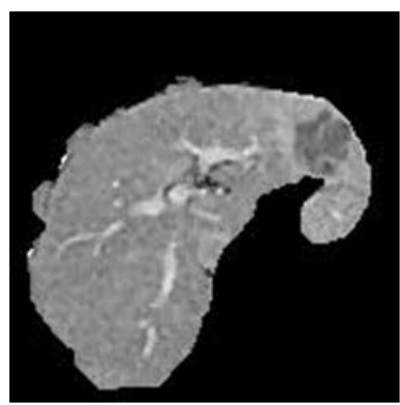

(f)

Figure 6: (a) Sample Input image, (b) Image after applying Median Filter, (c) Image obtained after applying sharpening filter, (d) Neural Network Classifier Output, (e) Refined output with post-processing, (f) Detected Liver in original image 


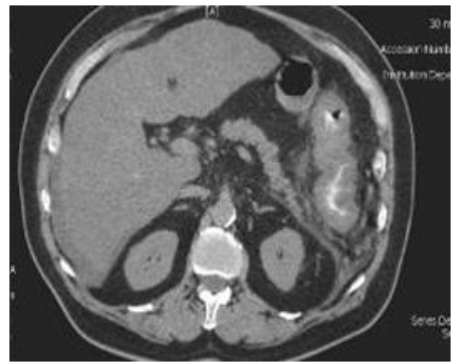

(a)

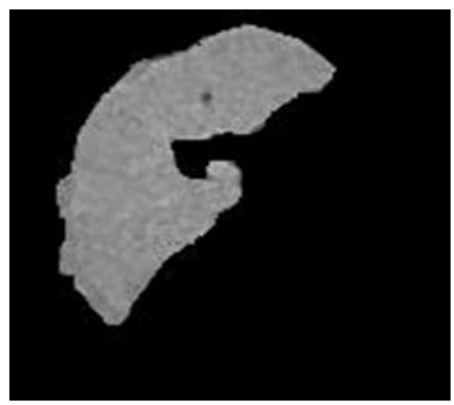

(d)

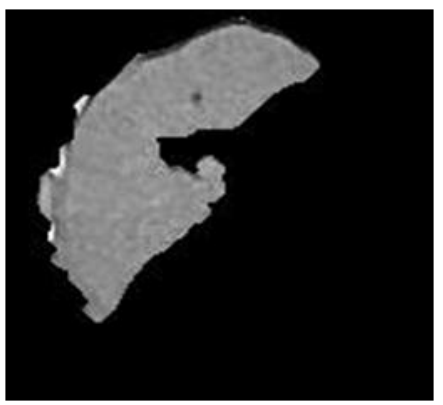

(g)

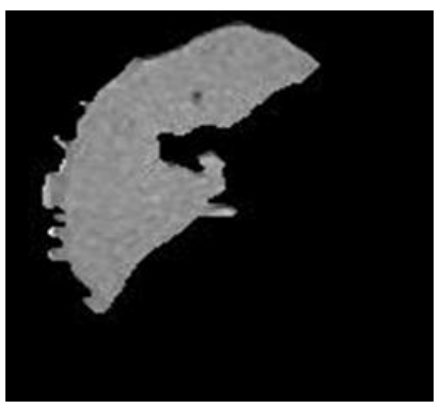

(j)

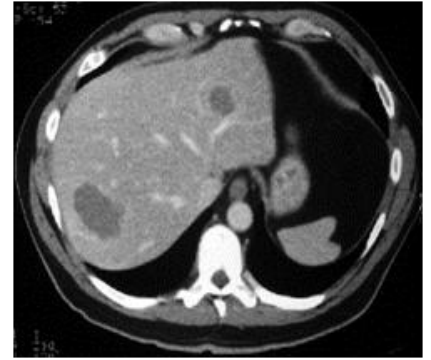

(b)

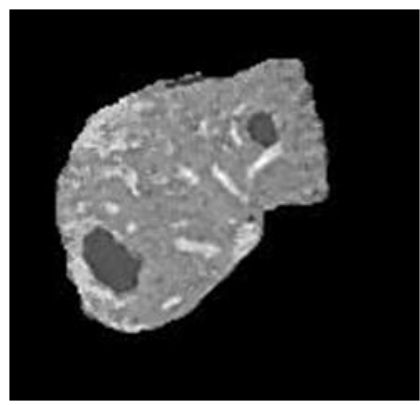

(e)

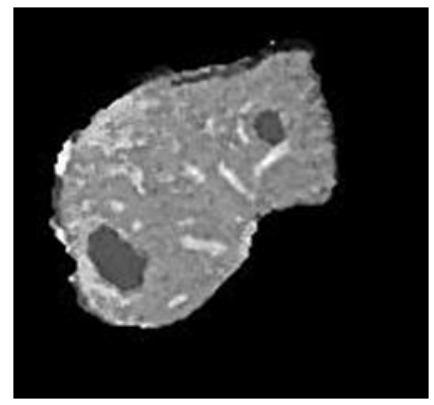

(h)

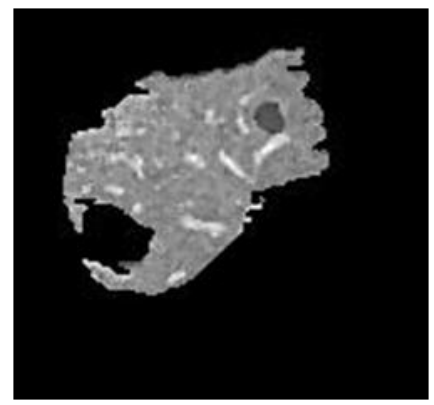

(k)

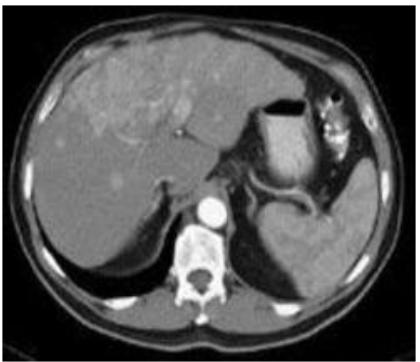

(c)

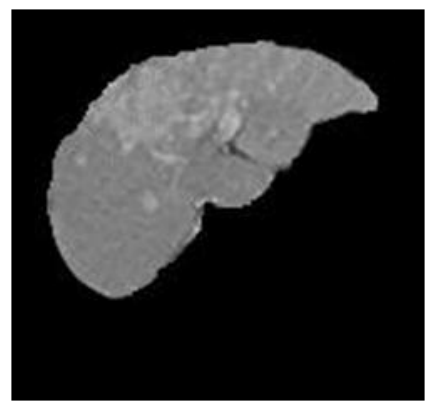

(f)

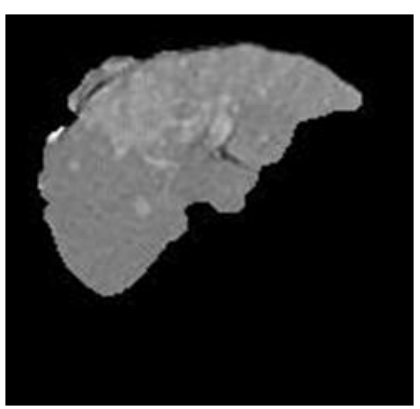

(i)

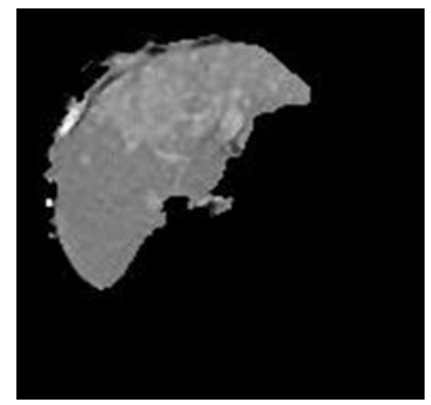

(1)

Figure 7: (a)-(c) Sample original CT images, (d)-(f) Results of Manual Segmentation, (g)-(i) Results Obtained through proposed Method (NN+GA), (j)-(l) Results Obtained Through NN Classification

For comparison, neural network based classification is used where the image is classified into liver and non-liver using neural network which is trained with complete feature matrix (not optimal). The proposed method (NN+GA) is applied on 10 CT images and Neural Network classification is applied on same images. Figure 7 shows results of three of these images. Liver image segmentation is a difficult task because abdominal images contain other organs also along with liver. Moreover other organs have similar intensity as of liver intensity. Because of these reasons, it becomes difficult to segment liver images using intensity based methods. Some feature based approach should be used. Further, extracted features should describe image properties well. For this purpose optimal features are chosen. For selecting optimal features, genetic algorithm combined with neural network has been utilized. Use of genetic algorithm, for feature selection, results in accurate classification of liver and non-liver pixels. Average accuracy obtained is $96.02 \%$ with the combination of genetic algorithm based feature selection and neural network classification. Further it reduces the time taken for 
classification process. Results of different parameters, calculated for both methods, are shown in Table 2. Different Parameters used are: true positive rate (TPR), true negative rate (TNR), false positive rate (FPR) and false negative rate (FNR).

Table 2. Quantitative Results

\begin{tabular}{|c|c|c|}
\hline Parameters & NN+GA & NN \\
\hline Average Accuracy & $96.02 \%$ & $87.64 \%$ \\
\hline Average True Positive Rate & $92.23 \%$ & $76.31 \%$ \\
\hline Average True Negative Rate & $97.52 \%$ & $92.37 \%$ \\
\hline Average False Positive Rate & 0.0248 & 0.0763 \\
\hline Average False Negative Rate & 0.0777 & 0.2369 \\
\hline
\end{tabular}

\section{CONCLUSION}

In this paper, a method for automatic liver detection is presented. Optimal features are extracted and used as input for neural network classifier. Texture features are utilized and in that histogram based features and wavelet transform based features are extracted. This feature set is very large and it may contain redundant features. So, for reducing this feature set, feature selection algorithm is used and optimal features are selected. For optimal feature selection genetic algorithm is used. Finally these features are given as input to neural network which is a pixel based classifier and it classifies pixels into liver and non-liver area. Results obtained from the proposed method prove its credibility for automatic detection of liver in liver images. The method shows that the application of genetic algorithm in combination with neural network gives more accurate results than simple neural network classification. Moreover, the liver detection time is greatly improved with this method.

\section{REFERENCES}

[1] Tsai, D., Tanahashi, N. 1994. Neural-network-based boundary detection of liver structure in CT images for $3 \mathrm{D}$ visualization. In Proceedings of IEEE international conference on neural networks. Vol. 6 (1). 3484-89.

[2] Koss, J.E., Newmann, F.D., Johnson, T.K., Kirch, D.L. 1999. Abdominal organ segmentation using texture transforms and a hopfield neural network. IEEE Trans Med Imaging. Vol. 18(7). 640-648.

[3] Haralick, R.M. 1979. Statistical and Structural Approaches to Texture, Proc. IEEE. Vol. 67(5). 786-806.

[4] Hussain S.A., Shigeru E. 2000. Use of neural network for feature Based Recognition Of Liver Region On CT Images. In proceedings of IEEE Signal Processing Society Workshop. Vol. 2. 831-840.
[5] Rafiee, A., Masoumi, H., Roosta, A. 2009. Using neural network for liver detection in abdominal MRI images. In International conference on signal and image processing applications IEEE. 21-26.

[6] Masoumi, H., Behrad, A., Ali Pourmina, M., Roosta, A. 2012. Automatic Liver Segmentation in MR images using an iterative watershed algorithm and artificial neural network. In Biomedical signal processing and control Elsevier. 429-437.

[7] Laine, A., Fan, J. 1993. Texture Classification by Wavelet Packet Signatures. IEEE Transactions Pattern Analysis and Machine Intelligence. Vol. 15(11). 11861191.

[8] Luo, S., Hu, Q., He, X., Li, J., Jin, J.S., Park, M. 2009. Automatic Liver Parenchyma Segmentation from Abdominal CT Images Using Support Vector Machines. International Conference on Complex Medical engineering, IEEE.

[9] Luo, S., Jin, J.S., Chalup, S.K., Qian, G. 2009. A Liver Segmentation Algorithm Based on Wavelets and Machine Learning. International Conference on Computational Intelligence and Natural Computing, IEEE.

[10] Lu, J., Wang, D., Shi, L., Ann Heng, P. 2012. Automatic Liver Segmentation in CT images based on Support Vector Machine In proceedings of the IEEE-EMBS International Conference on Biomedical and Health Informatics. 333-336.

[11] Chi, D., Zhao, Y., Li, M. 2010. Automatic Liver MR Image Segmentation with Self Organizing Map and Hierarchical Agglomerative Clustering Method. In 3rd International Congress on Image and signal processing, IEEE. 1333-1337.

[12] Gonzalez, Rafael C., Woods, Richard E. Digital Image Processing. Third Edition. Pearson Education.

[13] Materka, A., Strzelecki, M. Texture Analysis Methods A Review. Technical University of Lodz, Institute of Electronics, Brussels.

[14] Mallat, S. 1989. Multifrequency Channel Decomposition of Images and Wavelet Models, IEEE Trans. Acoustic, Speech and Signal Processing. Vol 37(12) 2091-2110.

[15] Sebe, N., Lew, M.S. 2000. Wavelet Based Texture Classification. In 15th International Conference on Pattern Recognition. Vol. 3. 947-950.

[16] Yang, J., Honavar, V. 1998. Feature Subset Selection using a Genetic Algorithm. Intelligent Systems and their Applications, IEEE. Vol. 13(2). 44-49.

[17] http://www.google.co.in/imghp?hl=en\&tab=wi 